\title{
Multifunctional and stable bone mimic proteinaceous matrix for bone tissue engineering
}

Jong-Eun Won ${ }^{a, b}$, Ye-Rang Yun ${ }^{a, b}$, Jun-Hyeog Jang ${ }^{c}$, Sung-Hee Yang ${ }^{a, b}$, Wojciech

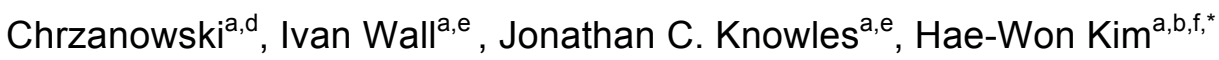

a Department of Nanobiomedical Science and BK21 PLUS NBM Global Research Center for Regenerative Medicine, Cheonan, 330-714, Dankook University, Republic of Korea

${ }^{b}$ Institute of Tissue Regeneration Engineering (ITREN), Dankook University, Cheonan, 330-714, Republic of Korea

${ }^{c}$ Department of Biochemistry, College of Medicine, Inha University, Incheon, Republic of Korea

${ }^{d}$ Faculty of Pharmacy, Pharmacy and Bank Building A15, University of Sydney NSW 2006, Australia

${ }^{\mathrm{e}}$ Division of Medicine, University College London, Rockefeller Building, 21 University Street, London, WC1E 6JJ, UK

${ }^{\mathrm{f}}$ Division of Biomaterials and Tissue Engineering, UCL Eastman Dental Institute, University College London, 256 Gray's Inn Road, London WC1X 8LD, UK

${ }^{9}$ Department of Biomaterials Science, School of Dentistry, Dankook University, Cheonan, 330-714, Republic of Korea

${ }^{*}$ Corresponding author: Prof. H.-W. Kim

E-mail) kimhw@dku.edu; Tel) +82 41550 3081; Fax) +82 415503085

For: Biomaterials 


\begin{abstract}
Biomaterial surface design with biomimetic proteins holds great promise for successful regeneration of tissues including bone. Here we report a novel proteinaceous hybrid matrix mimicking bone extracellular matrix that has multifunctional capacity to promote stem cell adhesion and osteogenesis with excellent stability. Osteocalcin-fibronectin fusion protein holding collagen binding domain was networked with fibrillar collagen, featuring bone extracellular matrix mimic, to provide multifunctional and structurally-stable biomatrices. The hybrid protein, integrated homogeneously with Col fibrillar networks, preserved structural stability over a month. Biological efficacy of the hybrid matrix was proven onto tethered surface of biopolymer porous scaffolds. Mesenchymal stem cells quickly anchored to the hybrid matrix, forming focal adhesions, and substantially conformed to cytoskeletal extensions, benefited from the fibronectin adhesive domains. Cells achieved high proliferative capacity to reach confluence rapidly and switched to a mature and osteogenic phenotype more effectively, resulting in greater osteogenic matrix syntheses and mineralization, driven by the engineered osteocalcin. The hybrid biomimetic matrix significantly improved in vivo bone formation in calvarial defects over 6 weeks. Based on the series of stimulated biological responses in vitro and in vivo the novel hybrid proteinaceous composition will be potentially useful as stem cell interfacing matrices for osteogenesis and bone regeneration.
\end{abstract}

Keywords: Bone mimetic; Protein engineering; Cell adhesion; Osteogenic differentiation; Multifunctional 


\section{Introduction}

Scaffolds with a capacity to repair and regenerate tissues including bone have undergone significant developments over the last few decades. In bone tissue engineering, scaffolds must satisfy a number of criteria including bioactivity, compatible surfaces for the control of cell fate, degradability within an appropriate time frame for substitution by neo-tissue, and three dimensional (3D) structures for inducing osteogenesis and angiogenesis [1].

The surface of scaffolds is considered of particular importance to optimize the biological processes including initial cellular recognition, tissue specific commitment and subsequent repair and regeneration. Mimicking the interface to the tissue of concern is by far the most promising strategy to appropriately regulate cellular and tissue reactions to ensure the success of scaffolds clinically. In fact, the extracellular matrix $(\mathrm{ECM})$ of the tissue provides the best micro-environmental conditions for the cells that are hosted and reside within it, and contain the necessary components required for tissue maintenance and recovery in the event of damage and disease [2].

Bone is a highly organized tissue that comprises the major part of human hard tissues, and performs both mechanical and biological functions. Bone ECM consists of collagen (Col) and also non-collagenous proteins such as fibronectin (FN), osteocalcin (OC), osteopontin (OPN), and bone sialoprotein (BSP) [3,4]. As the most abundant ECM component, Col is involved primarily in providing mechanical strength and aiding in the formation of mineralized bone matrix. Therefore, Col has been widely used in various clinical and tissue engineering applications. The use of Col as an interface of biomaterials has been researched, with results demonstrating that Col-surfaces enhance the attachment, proliferation, and differentiation of osteoblastic/progenitor cells in vitro, and subsequent bone formation in vivo [5-8].

Aside from the structural proteins, adhesive proteins such as fibronectin (FN), vitronectin, and laminin mediate cellular recognition of the underlying matrix through integrin signaling. $\mathrm{FN}$ is known to be a potent and dominant adhesive protein. FN contains three types of modules: I, II, and III, [9] and with respect to cell adhesion, the ninth and tenth domain of FN module III (FNIII ${ }_{9-10}$ ) contains Arg-Gly-Asp (RGD) and the synergic sequence Pro-His-Ser-Arg-Asn (PHSRN) is recognized to enhance cell adhesion, proliferation and differentiation of osteoblasts and progenitor cells $[10,11]$. However, the efficiency of the adhesive protein alone is not sufficient to stimulate differentiation and 
maturation (mineralization) of progenitor / stem cells in bone regenerative processes. In other words, some ECM proteins are critically involved in the differentiation and late stage mineralization of bone. Among else, $\mathrm{OC}$ is highlighted in this study. OC, the most abundant nanocollageneous protein in bone [4], accelerates mineral formation and growth due to its high affinity to the mineral crystals. In fact, OC has been recognized to be the key marker for late stage osteogenesis of progenitor / stem cells $[12,13]$.

We have recently synthesized a unique fusion protein, comprising OC and $\mathrm{FNIII}_{9-10}(\mathrm{OC}-\mathrm{FN}$ ), which was aimed at regulating sequential functions of mesenchymal stem cells both in the initial adhesion and late osteogenesis processes [13,14]. Based on this, here we design bone ECM mimic matrix where Col was introduced as the structural support and network of the OC-FN. In particular, we engineered OC-FN with collagen binding domain (CBD) to form a structurally hybridized ECM network with better physicochemical stability. Some engineered proteins containing CBD have already shown good performance with Col materials $[15,16]$. Moreover, we induced sufficient fibrillogenesis of Col, which is known to be a key elemental process to preserve Col stability and its native functions. The monomeric Col in solution form does not resemble the naturally occurring functional fibrillar form of collagen (fCol). The Col coating with poor fibrillar formation led to a lack of appropriate cellular responses, such as cell attachment and proliferation of osteoblasts $[6,17]$.

Collectively, the proteinaceous matrix constituted of Col-OC-FN is considered to resemble the native bone ECM and to favor stem cell functions including initial adhesion and late osteogenic differentiation while preserving the structural stability. In particular, here we coupled the protein matrix to a 3D porous scaffold made of an easily manufacturable and clinically approved synthetic biopolymer, polycaprolactone (PCL). The scaffold is widely used due to appropriate biodegradation rate and mechanical strength, but the lack of cell recognition site and tissue specific activity has been indicated as a major limitation for bone regenerative scaffolds $[18,19]$. Therefore, this approach of tailoring the biopolymer scaffold surface constructs with a bone supportive ECM matrix is a facile promising approach for development of next generation bone scaffolds. The processes to generate the protein hybridized scaffolds are described and their biological impacts are demonstrated in terms of mesenchymal stem cell responses in vitro and bone formation in vivo. 


\section{Materials and methods}

\subsection{Construction and purification of recombinant fusion protein, (CBD)OC-FN}

To construct the (CBD)OC-FN, OC-FN DNA was initially amplified by the CBD-OC-Sac forward primer, 5'-GGCGAGCTCACCAAAAAACCCTGCGTACCCTCGAGTACC TGTATCAATGGCTG-3, the pBAD-His reverse primer, 5'-ACGGCGTTTCACTTCTGAG T -3'. PCR was conducted for 30 cycles of $1 \mathrm{~min}$ at $55^{\circ} \mathrm{C}$ (annealing), $2 \mathrm{~min}$ at $72{ }^{\circ} \mathrm{C}$ (extension), and $1 \mathrm{~min}$ at $94{ }^{\circ} \mathrm{C}$ (denaturation). Amplified PCR products were digested with Sacl and HindIIII. After ligation into pBAD-HisA-OC-FN ${ }_{\text {III9-10 }}$ vector, pBAD-HisA-CBD-OC-FN ${ }_{\text {III9-10 }}$ constructs were produced. TOP10 E. coli, selected clones were grown in LB medium containing ampicillin overnight at $37^{\circ} \mathrm{C}$ after transformation. When the absorbance of cultures reached $0.6\left(A_{600}\right)$, induction was initiated with L-arabinose $(0.02 \% \mathrm{w} / \mathrm{v})$ and incubated at $20{ }^{\circ} \mathrm{C}$ for $6 \mathrm{~h}$. The bacterial pellets were harvested by centrifugation at $6,000 \times \mathrm{g}$ for $10 \mathrm{~min}$, lysed, and sonicated. A soluble extract obtained were centrifuged for $30 \mathrm{~min}$ at $14,000 \mathrm{x} \mathrm{g}$ in a refrigerated centrifuge and the supernatant was purified by affinity to a nickel-nitrilotriaceticacid resin (Invitrogen). To determine the protein, western blots were performed using a peroxidase conjugate of a monoclonal anti-polyhistidine antibody (SC-8036 HRP, SantaCruz). The degree of purification of the fusion protein was measured by carrying out sodium dodecyl sulfate-polyacrylamide gel electrophoresis (SDS-PAGE) $(\mathrm{Mw}=47 \mathrm{kDa})$ using SDS-PAGE at 10\% (v/v).

\subsection{Fibrillar formation of proteins and surface tailoring of scaffolds}

The collagen type I solution (60-30-810, First link Ltd.) was diluted in PBS and allowed for fibrillar formation upon immersion in incubator at elevated temperatures. To prepare Col-(CBD)OC-FN solutions, the (CBD)OC-FN fusion protein was added to the Col solution in PBS at different concentrations (fusion protrein: $\mathrm{Col}=1: 100,1: 200$, and 1:1000) before fibrillar formation. The degree of fibrillar formation of the protein solutions was characterized via turbidity test. The turbidity was monitored at $313 \mathrm{~nm}$ with varying incubation time, temperature and protein concentrations.

As the framework of scaffolds, macrochanneled PCL were fabricated using a RD (EzROBO3, Iwashita) as described previously [20. Scaffold was designed and controlled by CAD program. Prior to fabrication of the 3D scaffolds, PCL (MW 80,000, Sigma-Aldrich) were dissolved in 
acetone and then ball milled at $50{ }^{\circ} \mathrm{C}$. The temperature of the slurry was maintained via a thermojacket during the fabrication. The struts were formed in an ethanol bath for the solidification of the slurry via the needle $(0.33 \mathrm{~mm})$. The scaffolds were designed as size of $9 \times 9 \mathrm{~mm}$ and with 6 layers.

For surface of PCL scaffolds was then tailored with the protein solutions. For this, the PCL scaffolds were first hydrolyzed and activated with a cross-linker (EDC-NHS). The pretreated scaffold was then immersed into the different protein solutions for predetermined time period at room temperature. The protein adherence to the surface was assessed by the detection of Col using Sirius Red staining method. Direct red 80 (365548, Sigma) dissolved in saturated picric acid was added to the scaffolds and then left for $1 \mathrm{~h}$ at room temperature. The stained samples were washed completely with $0.01 \mathrm{~N} \mathrm{HCl}$. The surface-adhered Col was quantified by elution with $200 \mu \mathrm{l}$ of $0.5 \mathrm{M} \mathrm{NaOH}$. The eluted solution was transferred to 96 -well plates, and the optical density was measured at $540 \mathrm{~nm}$. The values were converted to a Col concentration when referenced to a standard curve of Col solution [6]. The optical density (OD) by Sirius red assay showed a linear relationship with Col concentration; $O D=1.643 \times$ Col concentration $+0.0269\left(R^{2}=0.99\right)$.

The (CBD)OC-FN distribution within Col matrix was examined by TEM after dropping the protein solution on a Cu-grit, followed by staining with Au nanoparticles (ab154873, abcam) conjugated with OC-antibody. The (CBD)OC-FN within the Col-bound scaffolds was also directly visualized by immunostaining against OC. The scaffolds tailored with the proteins were put into OC antibody (1:200, SC-365797, Santacruz). After treatment with a blocking buffer to block nonspecific binding (5\% skim milk), anti-mouse-Alexa Fluor 555 (1:500, A21422, Invitrogen) was used for conjugation with primary antibody. The fluorescence image was obtained by confocal laser scanning microscopy (CLSM, Carl Zeiss 510L, Germany). The intensity from the fluorescence image, corresponding to the quantity of fusion protein in the hybrid protein network, was measured using image $\mathrm{J}$ program $(\mathrm{NIH})$.

An indirect ELISA protocol was also used for the quantification of the fusion protein. After the adhesion, the non-bound protein solution was transferred into the 96 well of ELISA plates and incubated overnight at $4{ }^{\circ} \mathrm{C}$. After blocking, the His-probe antibody (1:500, SC-8036, Santacruz) and secondary antibody (1:500, SC-2005, Santacruz) were bound with the His-tagged protein. As the substrate, 1-Step Turbo TMB-ELISA solution (34022, ThermoScientific) was used. After color change, the reaction was stopped using $2 \mathrm{~N}$ sulfuric acid. The absorbance was read at $450 \mathrm{~nm}$ using a 
microplate reader (SpectraMax M2e, Molecular Devices).The optical density was calibrated using protein standard curve. All values were normalized to the value for collagen.

The surface-adhered morphology of the protein-tailored scaffolds was observed by scanning electron microscopy (SEM; JSM-6510, JEOL, Japan) and high resolution SEM (HR-SEM; MIRAII LMH, TESCAN, Czech Republic). The nanotopography of the proteins tethered on the scaffolds was examined by molecular force probe (MFP-3D-Bio, AsylumResearch, California, USA). To test wetting ability, samples were prepared by aligning strains in direct contacts by the RD machine. The water contact angle was measured using the sessile drop method. The $\mathrm{pH}$ of the Col solution was assessed to determine the effect of fibril formation using a $\mathrm{pH}$ meter (Thermo Scientific Orion 3 star, ColeParmer, USA).

\subsection{Rat mesenchymal stem cells (rMSCs) and the adhesion and proliferation assays}

The rat MSCs were collected as detailed previously [21]. In brief, the bone marrow was harvested from femora and tibiae of adult rats (age 4 weeks, Korean) and was treated with collagenase type I. The suspension was sieved and centrifuged, and then the collected cells were cultured in total culture medium that is a-MEM supplemented with $10 \%$ fetal bovine serum and $1 \%$ penicillin/streptomycinin humidified atmosphere of $95 \%$ air and $5 \% \mathrm{CO}_{2}$ at $37{ }^{\circ} \mathrm{C}$. Non-adherent cells were removed by medium exchange and the cells were maintained to reach near confluence forming a monolayer and the passage 3 was used for further evaluations.

Prior to seeding cells, the scaffolds were sterilized using $70 \%$ ethanol and UV light. The scaffold was put in the well plate and $1 \times 10^{5}$ cells in $100 \mu$ l culture medium were seeded on the scaffolds. After $1 \mathrm{~h}$, the samples were transferred to 48 well plate and $800 \mu \mathrm{l}$ fresh medium was then added into well. The medium was changed every other day.

The cell adhesion and viability were determined by MTS assay, which is based on reduction of tetrazolium salt by dehydrogenases of mitochondria in cell to produce purple formazan crystals. The cultured cells on the scaffold were washed with PBS and transfer to new culture plate then incubated with $400 \mu \mathrm{l}$ MTS solution, which was mixed as ratio of $10 \%$ CellTiter 96 AQueous One Solution (Promega) in medium, for $3 \mathrm{~h}$ at $37{ }^{\circ} \mathrm{C}$. The $200 \mu \mathrm{l}$ reactant was transferred into 96 well plates and the absorbance was measured at $590 \mathrm{~nm}$.

To observe the shape and morphology of rMSCs adhered onto the scaffolds, 
immunocytochemistry was carried out. After samples were fixed with $4 \%$ paraformaldehyde, the cells were permeabilized with $0.2 \%$ TritonX100. Anti-vinculin antibody (1:200 dilution, ab18058, abcam) was used as primary antibody. As the secondary antibody, fluorescein isothiocynate (FITC)-labeled antibody (sc-2010, SantaCruz) was used with phalloidin (A22283, Invitrogen) to stain F-actin and the nuclei were stained with DAPI (P36935, Invitrogen). Fluorescence images were taken by CLSM using $z$-stack mode. To analyze the size of cells spreading on the scaffolds, Zen 2009 image program was used.

The expression of genes involved in the initial adhesion of the rMSCs was observed using reverse transcription polymerase chain reaction (RT-PCR). At predetermined time points, the cultured cells were harvested and RNA extraction was followed using the QiagenRNeasy mini kit instruction (Qiagen). The isolated RNA quantified was then converted to complementary DNA (cDNA) with first strand cDNA synthesis kit (PrimeScript RT reagent Kit, Bioneer, Korea). As the initial adhesion marker, the adhesive protein paxillin was detected. The primer was designed according to published cDNA sequences in GenBank. Glyceraldehyde phosphate dehydrogenase (GAPDH) was used as the housekeeping gene.

To observe the morphology of cells on the scaffolds, the samples were fixed and permeabilized. For fluorescence image, the rMSCs on the scaffold were stained with phalloidin and DAPI. For SEM image, the samples were dehydrated with a gradient series of ethanol, treated with hexamethyldisilazane, and then coated with platinum.

\subsection{Osteogenic differentiation of MSCs and mineralization}

To confirm the osteogenic ability of the rMSCs grown on the protein-tailored scaffolds, the osteogenic medium, which is total culturing medium plus $50 \mu \mathrm{g} / \mathrm{ml}$ ascorbic acid, $10 \mathrm{mM} \beta$-glycerol phosphate, and $10 \mathrm{nM}$ dexamethasone, was added and the cells were incubated for periods of up to 28 days.

Alkaline phosphatase (ALP) activity, as an early marker of osteoblastic differentiation, was first determined. After the cells were lysed with RIPA buffer, the lysate solutions were reacted at $37^{\circ} \mathrm{C}$ for $3 \mathrm{~h}$ with a reagent, composed of a mixture of $20 \mathrm{mM}$ p-nitro phenol phosphate, $1.5 \mathrm{M}$ 2-amino2methyl-1-propanol, and $1.0 \mathrm{mM} \mathrm{MgCl}_{2}$ at 1:1:1 ratio. After incubation, the samples were set on ice and the reaction stopped with $100 \mu \mathrm{l} 1 \mathrm{M} \mathrm{NaOH}$. The absorbance was measured at $405 \mathrm{~nm}$ and the amount of ALP was calculated as the p-nitrophenol standard curve. Finally, ALP activity was 
expressed as the value per unit double strand DNA (dsDNA) concentration which was measured using a Picogreen kit (Invitrogen).

Cellular mineralization, a final stage of osteogenesis, was evaluated by Alizarin red $S$ (ARS) staining method. The ARS dye was dissolved in distilled water and the $\mathrm{pH}$ was adjusted to $4.2-4.4$ using $10 \%(\mathrm{v} / \mathrm{v})$ ammonium hydroxide. The ARS solution was added into each sample and left for 5 min to react with calcium, after which the sample was washed completely with distilled water and then dried. To extract of the stained calcium, 10\% cetylpyridinium chloride (CPC) was prepared and $400 \mu \mathrm{l} \mathrm{CPC}$ was added in each sample. The value was expressed by the optical density (OD) at 405 $\mathrm{nm}$. The mineral elements ( $\mathrm{Ca}$ and $\mathrm{P}$ ) were detected by energy dispersive spectroscopy (EDS, Oxford), in conjunction with SEM.

\subsection{In vivo implantation in rat calvarium defect}

Ten weeks old male Sprague-Dawley rats (DaehanBiolink Co., Ltd, Chungbuk, Korea) were used in the in vivo experiments. Animals were maintained in a $12 \mathrm{~h}$ light/dark cycle (lights on from 8:00 to $20: 00)$, with relative humidity $(30-70 \%)$ and temperature $\left(20-24{ }^{\circ} \mathrm{C}\right)$-controlled environment. The rats were allowed normal diet and water ad libitum. The experimental protocol for all animal care and use in this study was reviewed and approved in accordance with guidelines established by the Animal Care and Use Committee, Dankook University, Cheonan, Korea.

General anesthesia was induced by an intramuscular injection a mixture of ketamine (80 $\mathrm{mg} / \mathrm{kg})$ and xylazine $(10 \mathrm{mg} / \mathrm{kg})$. The animals were maintained under general anesthesia during the entire surgical procedure. The scaffolds with diameter of $5 \mathrm{~mm}$ were prepared sterile prior to use. The hair over the skin on the dorsal region of the cranium was shaved and the region was aseptically prepared using povidone and $70 \%$ ethanol for surgery. A linear sagittal midline skin incision was made over the calvarium with a \#10 surgical blade. Full thickness flap of the skin and periosteum was elevated to obtain sufficient surgical field for the drilling. Two $5 \mathrm{~mm}$ diameter critically sized circular full-thickness calvarial bone defect were created in each rat, at the centre of each parietal bone. To create the defects without overheating of the bone edges, a trephine bur was used under constant irrigation with sterile saline solution, without damaging the underlying sagittal sinus and dura matter.

The defects were randomly allocated into five groups; protein-free scaffold (PCL), protein 
tailored scaffolds with different (CBD)OC-FN fusion protein content of 0 (Col), $1 \mu \mathrm{g}$ (1OCFN), $10 \mu \mathrm{g}$ (10OCFN), and $100 \mu \mathrm{g}(100 \mathrm{OCFN})$. Total 15 animals (2 defects per animal) were used where six samples for each group were distributed into the defects. After the implantation, subcutaneous tissue was closed with absorbable materials (4-0 Vicryl, Ethicon, Norderstedt, Germany), and skin incisions were sutured with non-absorbable materials (4-0Dafilon, B. Braun Melsungen AG, Melsungen, Germany).

The animals were housed individually in cages and monitored during the experimental time for signs of infection, inflammation, and any adverse reaction by visual observation. Six weeks following operation, the animals were sacrificed to collect the samples. Skin was dissected, and the tissues including the surgical sites and its surrounding tissues were harvested along with surrounding bone. The specimens were fixed in $10 \%$ buffered neutralized formalin for $24 \mathrm{~h}$ at room temperature and prepared for micro-CT $(\mu \mathrm{CT})$ analysis and histology.

\subsection{Quantitative and qualitative assessments of bone formation}

Following fixation, the $\mu \mathrm{CT}$ images were performed to visualize the samples and measure defect closure for quantitative image analysis of new bone formation. The harvested specimens were scanned using $\mu \mathrm{CT}$ scanner (Skyscan 1176; Skyscan, Aartselaar, Belgium) with a camera pixel size of $12.56 \mu \mathrm{m}$, and a frame averaging of three was employed together with a filter of $1 \mathrm{~mm}$ aluminum, a rotation step of $0.5^{\circ}$, the rotation angle was $180^{\circ}$. The X-ray tube voltage was $65 \mathrm{kV}$ and the current was $385 \mu \mathrm{A}$, with an exposure time of $279 \mathrm{~ms}$. A cylindrical region of interest (ROI) was positioned over the center of the single defect, enclosed all the new bone within the defect site. Percent bone volume of newly formed bone within the ROI was measured and 3D $\mu \mathrm{CT}$ images were reconstructed through a computer analysis program, CTAn (Skyscan). The percentage of bone volume (\%), bone surface $(\mathrm{mm})$, and bone surface density $(1 / \mathrm{mm})$ of the newly formed bone within each ROI were measured by assigning a threshold for total bone content (including trabecular and cortical bone).

After $\mu \mathrm{CT}$ imaging, the harvested samples were prepared for histological analysis. For this, fixed specimens were decalcified with RapidCal ${ }^{\top \mathrm{M}}$ solution (BBC Chemical Co., Stanwood, WA, USA), dehydrated through a series of ethanol solutions of increasing concentration (from 70 to $100 \%$ ) and 
subsequently embedded in paraffin. Approximately $5 \mu \mathrm{m}$ thick coronal sections were prepared at the central region of the circular defects using a microtome (RM2245, Leica, Germany) and defined as the representative histology. The slides were stained with hematoxylin \& eosin (H\&E) with routine techniques and examined using a light microscope (IX71, Olympus, Tokyo, Japan). Results of new bone formation within the defect were taken under $40 \mathrm{x}$ magnification.

\subsection{Statistical analysis}

The data were presented as the mean and standard deviation (SD). The statistical difference between groups was carried out by analysis of variance (ANOVA), and the significance level was considered at $p<0.05$.

\section{Results and discussion}

\subsection{Protein mimics and engineering on scaffolds}

The schematic image of the currently used recombinant (CBD)OC-FN fusion protein is shown in Fig. 1a, and Western blot analysis revealed a single protein band at around $50 \mathrm{kDa}$ (Fig. 1b). The FN domain was structured to be tenth and ninth type III, which has a specific region of RGD and PHSRN respectively for cell adhesion. On the other hand, the $\mathrm{OC}$, holding carboxyglutamate residues, has a high affinity for calcium ions thus potentially contributing to the later osteogenic differentiation and mineralization processes. Bi-functionality of the OC-FN fusion protein has already been shown with respect to initial cell adhesion and osteogenic differentiation $[13,14,22]$. The CBD linked to the OC-FN fusion protein has specific affinity to Col matrix, which is the major structural component of the current engineered ECM. The TKKTLRT short peptide sequence contained in CBD has previously been used to specifically bind to Col matrices $[15,23]$. We thus consider that the proteinaceous matrix of hybrid $\mathrm{Col}+(\mathrm{CBD}) \mathrm{OC}-\mathrm{FN}$, engineered as a surrogate bone ECM, will provide proper substrate conditions for cellular growth and osteogenesis.

In particular, we paid attention to fibrillar formation of Col structure as this is the naturally 
occurring functional structure, which plays essential roles in triggering cellular responses and preserving structural integrity $[6,17]$. Generally, Col solutions are in acidic soluble form with monomeric tropocollagen state, necessitating optimal fibrillar formation. The degree of fibrillar formation was thus assessed by the change in turbidity of the solutions with time, set in different conditions such as variation in temperature $\left(25\right.$ and $\left.37^{\circ} \mathrm{C}\right)$ and Col concentration in PBS $(5,10$ and 20\%) [24-26]. Turbidity generally increased with time after some lag period, and then appeared to saturate within approximately $10 \mathrm{~h}$ (Fig. 1c). In the test at $25^{\circ} \mathrm{C}$ with varying Col concentration, the turbidity increase was the most rapid and the OD value was the highest at $10 \%$ Col $(10 \%>5 \%>$ $20 \%$ ), although all concentrations profiled similar turbidity kinetics. With $10 \%$ Col, the temperature was again varied to $37^{\circ} \mathrm{C}$, where the turbidity increase was abrupt as early as within few minutes and then saturated with the maximal level lower than that measured at $25^{\circ} \mathrm{C}$. In the same context, Wood et al. also reported similar results at both 25 and $37^{\circ} \mathrm{C}$ [24]. In the Col assembly process, the lag phase is critical for the nucleation of monomers and subsequent fibrillar formation with sufficient size. Based on these results, the assembly of $10 \% \mathrm{Col}$ in PBS at $25{ }^{\circ} \mathrm{C}$ was selected as the optical condition. We next tested fibrillar formation of the Col+(CBD)OC-FN hybrid protein in a similar way to pure Col while varying the fusion protein concentrations (OC-FN per $200 \mu \mathrm{g} \mathrm{Col}=0.1,1$ and $2 \mu \mathrm{g}$ ). The turbidity change behaved similarly for all protein compositions with only slight decrease with increasing OC-FN concentration (Fig. 1d).

The hybrid protein, Col+(CBD)OC-FN, was primarily used to tailor the surface of 3D porous scaffolds, aiming to control the biointerface between scaffold and cells. 3D macrochanneled PCL scaffolds designed by robotic dispensing were used as model polymeric supports to determine the effects of engineered hybrid protein matrix in vitro and in vivo. After surface activation of the polymeric scaffold, a solution-based protein tethering under gentle agitation gave uniform protein distribution throughout the scaffold's 3D macroporous network (Fig. 2a including inset red-colored scaffold image stained by Sirius red). Protein matrix covered the surface almost completely. Well-developed fibrillar Col structure was revealed on high resolution SEM images (Fig. 2b,c). Furthermore, the molecular force probe image revealed 3D topology of the hybrid protein on the scaffold, containing $67 \mathrm{~nm} D$ periodic patterns, which is typical of well-assembled fibrillar Col (Fig. 2d). The surface roughness change with the protein tailoring was found to be insignificant by the molecular force probing (data not 
shown). The existence of OC-FN in the hybrid network was, however, not easily recognized in the optical and electron microscopy. To reveal the OC-FN, the hybrid protein (1OCFN) prepared directly on Cu-grit was immunostained with Au nanoparticles ( $40 \mathrm{~nm}$ size) against $\mathrm{OC}$ and then observed by TEM (Fig. 2e). A number of dark spots were well visualized, evenly distributed throughout the matrix, which are assigned to be OC-FN, and the average density of the dots was $50 / \mathrm{mm}^{2}$ with single dot occupying approximately $20 \mathrm{~nm}^{2}$. When we consider the concentration ratio of Col and OC-FN pooled in the hybrid network, such a density level was calculated to be reasonable.

The hybrid protein structured on the PCL scaffolds was quantified by Sirius red staining. The quantity of Col on the scaffold was around $130 \mu \mathrm{g}$ without regard to the existence of OC-FN, which corresponds to $65 \%$ of the Col used initially $(200 \mu \mathrm{g})$ (Fig. 3a). Furthermore, the quantity of OC-FN present on the Col network was analyzed by immunostaining with OC primary / HRP-conjugated secondary antibodies (Fig. 3b). Interestingly, the OC-FN present on the tethered Col network ( 800 $\mathrm{ng})$ was higher than that on the solution Col state $(\sim 500 \mathrm{ng})$ by 1.6 times difference. The Col+OC-FN protein matrix integrated on PCL scaffold surface was further analyzed the degradation stability. The Sirius red assay of the protein remaining on the surface enabled us to characterize degradation of Col with time measured for up to 28 days (Fig. 3c). Col degradation with time was obvious; $\sim 30 \%$ for the $1^{\text {st }}$ week, up to $45 \%$ during 2 weeks and up to $60 \%$ during 4 weeks. While the Col showed on-going degradation with time, the degradation of OC-FN in the Col was monitored by the fluorescence images with time (Fig. 3d). Interestingly, the fluorescence intensity changed very little (based on both images and quantification), preserving the initial fluorescence level prior to degradation test. The OCFN is considered to tightly bind to Col network without a premature release from the structure, being distributed at an almost equal quantity with respect to Col, which presumably preserve the fluorescence signals in the Col matrix, which remained in large amounts on the scaffold covering the surface completely.

\subsection{Cell adhesion, spreading and proliferation}

The cellular responses to the Col+(CBD)OC-FN protein interfaced scaffolds were investigated using rMSCs. Prior to in-depth cell tests, the effect of (CBD)OC-FN concentration on cell adhesion was first screened on a tissue culture plastic during $4 \mathrm{~h}$ by means of MTS assay. Cell adhesion gradually 
increased with increasing protein concentration up to $1 \mu \mathrm{g}$, after which showing a plateau up to $10 \mu \mathrm{g}$. Based on this, we used the 1OCFN as the representative of hybrid protein matrix, and Col matrix and bare PCL for comparison groups. In Fig. 4a, the cellular adhesion was monitored at the varying time points $(2,4$, and $8 \mathrm{~h}$ ). While cell adhesion was largely limited on PCL, Col tailoring substantially increased the cell adhesion level. Furthermore, 10CFN group improved the cell adhesion more significantly. This was particularly evident at the very initial period of cellular adhesion as early as $2 \mathrm{~h}$, where a big difference in the adhesive level between the groups was seen. The mRNA expression of paxillin (PAX), a representative of adhesive proteins involved in the early cell adhesion processes, was also significantly stimulated by the engineered proteins at the early periods tested (1OCFN > Col $>$ PCL) (Fig. 4b).

The initial cell adhesion and spreading behaviors were visualized by CLSM after coimmunostaining of nuclei with DAPI (blue), F-actins with phalloidin (red) as well as vinculin (green) (Fig. 5a). Only few cells were found on PCL, where the F-actins and vinculin stains were also in very limited area. On Col-surfaced scaffold, more cells were found and the cells extended better with a spreading morphology and with more intensified vinculin signals at the periphery, particularly at $4 \mathrm{~h}$. On 1OCFN-tethered surface, the cellular extension was substantial as early as $2 \mathrm{~h}$, with similar patterns to those noticed in Col matrix at $4 \mathrm{~h}$. While the cell morphology was rather rounded at $2 \mathrm{~h}$ in 1OCFN (vs. $4 \mathrm{~h}$ in case of $\mathrm{Col}$ ), it became irregular with more lamellar extensions in certain directions, possibly due to the shift from filopodia to lamellipodia extensions $[27,28]$. Furthermore, whilst the vinculin signals were mostly obvious at the periphery at $2 \mathrm{~h}$, the signals became diffused after $4 \mathrm{~h}$, signifying possible change in spatial distribution of adhesion complex proteins, the phenomenon often known as when cells underwent substantial spreading from initial focal contacts. The cell spreading behavior was subsequently quantified to define cell spreading area and perimeter (Fig. 5b). Compared to pure PCL scaffolds, the protein-tailored scaffolds showed significantly higher values of both indices and the 1OCFN group showed the higher values. Furthermore, while there was little change with culture time in both indices in the PCL scaffold, there was substantial increase with time for both protein-surfaced scaffolds. At $4 \mathrm{~h}$, the cell perimeters on Col and 1OCFN were 3.9- and 5.2fold higher, and the areas were 4.6- and 8.6-fold higher, than those on PCL. In comparing between Col and 1OCFN, the cell perimeter and area were 1.3- and 2.8-fold difference, respectively. 
Taking all the cellular adhesion studies, rMSCs were stimulated to better recognize the hybrid protein matrix, with significantly higher cellular anchorage, and then conformed to spread more quickly with expressions of focal adhesive molecules at the gene and protein levels and with apparent cytoskeleton extensions. While the Col, with its adhesive ligands, also substantially improved those adhesion behaviors of rMSCs to the cell-ligand free PCL surface, the involvement of FN with the III910 adhesive domains invoked significant improvement of rMSCs adhesion behaviors. In fact, FN, among other adhesive proteins including Col, vitronectin and laminin, has shown the greatest potential for MSCs adhesion process through signaling of integrins such as $\alpha v \beta 3$ and $\alpha 5 \beta 1$ [29].

After confirming the stimulatory effects on rMSCs adhesion and spreading, we next examined the cell proliferative behaviors on the protein matrices. The MTS optical density, corresponding to cell viability, was plotted at each culture period for up to 14 days (Fig. 6a). The difference in MTS level, noticed as early as day 1, was preserved up to the test period. The MTS increment with time was also plotted to examine the cell proliferative potential (Fig. 6b). In Col and 1OCFN groups, the proliferative potential was relatively higher at initial periods $(<7$ days), but decreased as the culture time increased. On the other hand, in the PCL group, the proliferative potential was low initially ( $<7$ days) and then became increased substantially after 7 days. It is thus considered that when the protein matrix was interfaced, the rMSCs acquired proliferative capacity more quickly to reach confluence and subsequently switched to next cellular processes involving prominently differentiation. ${ }^{12}$ The representative morphologies of cells grown on the $10 C F N$-tailored scaffolds were observed using SEM and CLSM (Fig. 6c-e). At 14 days cells already reached confluence (Fig. 6c), covering the scaffold surface almost completely in a uniform way, as examined by a cross-sectioned SEM image (Fig. 6d), moreover, confocal image revealed a number of cytoskeletal processed networks and formation of thick cell-ECM constructs throughout the scaffolds, an indication that cells secreted a bunch of matrix molecules at this culture period (Fig. 6e).

\subsection{Osteogenic differentiation}

After confirming stimulation of rMSC responses including initial adhesion, spreading and timesequenced proliferation, we next examined the osteogenic differentiation of cells. Alkaline phosphatase (ALP), which is regarded as an early marker of osteogenesis, was substantially 
expressed during the culture from 7 to 14 days, with significantly higher levels on Col than on PCL as well as those on 1OCFN than on Col. The ALP level from 14 to 21 days was strongly reduced in both Col and 1OCFN, which however, was slightly increased in PCL (Fig. 7a). Because ALP is representative of early osteogenic marker, the rMSCs at the relatively prolonged culture over 14 days will reduce ALP activity and predominantly secret other ECMs that are required at much later stage of osteogenesis and mineralization, demonstrating the rMSCs grown on the protein-tailored scaffolds underwent substantial degree of osteogenic differentiation, expressing ALP earlier in culture.

Lastly, the cellular mineralization was assessed at prolonged culture periods of up to 28 days by Alizarin red staining (ARS) (Fig. 7b). From the ARS optical images, the mineral deposit on the 1OCFN-tailored scaffolds revealed much clearer red staining. From quantification of the stained extracts, the mineralization level substantially increased with culture period on both Col and 1OCFNtailored groups (even better on 1OCFN) than on PCL, which showed very limited change with time. As a consequence, significantly higher mineralization was recorded in the sequence: 1OCFN > Col > PCL. To confirm the atomic composition of the mineral deposits, we gathered the deposits and analyzed with EDS equipped with TEM (Fig. 7c). The EDS spectra of cellular deposits in 1OCFN group revealed high intensities of $\mathrm{Ca}$ and $\mathrm{P}$ peaks, with a $\mathrm{Ca} / \mathrm{P}$ ratio of 1.507 , a value similar to but lower than the stoichiometry of hydroxyapatite. The appearance of $S$ might be cellular components or organic ECMs additionally present on the EDS sample. The mineralization data demonstrates well the potential of the OCFN in the finalizing stage of MSCs osteogenesis. In this mineralization phase, the presence of ECM plays critical roles in capturing and precipitating calcium and phosphate ions. Other than Col, which is exogenous and rich in the matrix, many endogenous ECM proteins that were secreted by the cells, such as OP and BSP as revealed in our study as well, should affect the cellular mineralization process. Among other ECM molecules, the OC, used as the key exogenous moiety in the hybrid matrix, has been a potent calcium binding protein, critically involved in accelerating cellular mineralization. ${ }^{29}$ Taken together the in vitro findings, the hybrid proteinaceous matrix of $\mathrm{Col}+(\mathrm{CBD}) \mathrm{OC}-\mathrm{FN}$ stimulated rMSCs in the series of responses from the early adhesion phase to late osteogenesis and mineralization.

\subsection{In vivo bone formation}

The in vivo effects of the hybrid protein tailoring of the scaffolds on bone regeneration were assayed 
using a rat calvarial defect model (as shown the in vivo model in Fig. 8a). The in vitro experiments clearly showed the effectiveness of the (CBD)OC-FN concentration $1 \mu \mathrm{g}$. Therefore, we carried out the in vivo study with this sample group, which resulted in very marginal improvement in bone regeneration. We considered the dose needed in the in vivo condition might be higher than that in cellular studies in vitro. We thus redesigned the in vivo experiments with increasing protein concentrations at 10 and $100 \mu \mathrm{g}$. After implantation of scaffolds (PCL, Col, 1OCFN, 10OCFN, and 100 OCFN) for 6 weeks, the degree of bone growth was assessed by using $\mu C T$. Fig. $8 \mathbf{b}$ shows the representative planar views of the $\mu \mathrm{CT}$-constructed images of all groups. There was little bone formation within the defect region in the negative control, confirming the critical-sized defect of the model. Within the pure PCL scaffold, some level of new bone was formed, suggesting the osteoconductive role of porous scaffolds. When Col-surfaced scaffold was used, bone formation appeared to be increased. When the fusion protein OCFN was hybridized with Col, the scaffolds increased the bone formation, and particularly with 1000CFN almost all the defect was filled with newly formed bone on planar view. The 3D de novo bone structure was further analyzed by $\mu \mathrm{CT}$ software to characterize bone volume, bone surface and bone surface density (Fig. 8c). The bone volume was significantly increased with the Col-tailoring, and the involvement of OCFN in Col further increased in concentration-dependent manner; significant improvements were noticed with 100CFN and $100 \mathrm{OCFN}$, whilst not with 1OCFN. The bone surface and surface density were also significantly improved with $1000 \mathrm{CFN}$ with respect to Col, which however, was not recognized with 1OCFN and 10OCFN. While the bone volume means the quantity of regenerated bone, the bone surface density reflects the quality of regenerated bone in the entire bone-defect region based on the information of bone volume and bone surface. It is thus clear that the in vivo effects of OCFN in Col matrix were more obvious, not only in the quantity but in the quality also, when the concentration was over $10 \mu \mathrm{g}$. This concentration range was much higher than the concentration $(1 \mu \mathrm{g})$ effective in vitro on rMSCs responses. This is an important consideration because translating from simple in vitro models to physiologic systems places a more severe set of demands on a system and so dosage or drug concentration will need to be changed accordingly. The more dynamic and harsh in vivo conditions compared to confined and simple in vitro cell culture conditions might require higher doses of therapeutic molecules that are effective in recruiting progenitor / stem cells, anchoring them, stimulating them to secret matrix molecules, before subsequent mineralization. It should be 
underscored though that with the higher in vivo dose, the stimulatory role of OCFN+Col matrix in bone regeneration was noteworthy; compared with $\mathrm{PCL}$ or Col groups, the increase in bone volume was from $25-30 \%$ to $50-60 \%$ (2-fold) and that in bone surface density was from $4-4.5 \mathrm{~mm}^{-1}$ to $6-7 \mathrm{~mm}^{-1}$ (1.5 fold).

Histological analyses of the tissue samples were also carried out with H\&E staining after decalcification. The histological images support well the $\mu \mathrm{CT}$ results. Complete bridging of the defect gap through bone regeneration was only observed in 1000CFN group while partial limited bone regenerations were noticed in other scaffolding groups, with no bone-like tissues in the blank control (Fig. 9a). A closer examination of the histological images gave the information on tissue responses and bone quality (Fig. 9b). The scaffold surface was in direct contact with the newly formed bone without interposition of connective tissues. The newly formed bone in the 1000CFN group was largely mature and compact with a lining of osteoblasts, confirming the lamellar-structured bone, and some areas were comprised of loosely organized woven bone matrix, which would possibly be replaced with lamellar bone by remodeling process with time. Moreover, bone marrows were only formed in the new bone matrix of the 1000CFN group. This histological feature of bone quality also demonstrates the effective potential of the engineered protein matrix to create rapid bone formation and its subsequent remodeling during the 6 weeks of implantation period to conform to matured lamella structure.

\section{Summarizing scheme}

In summarizing the present findings, we illustrate a scheme of the possible series of reactions involved in the stimulatory roles played by the mimetic hybrid Col+(CBD)OC-FN matrices (Fig. 10). MSCs specifically recognize the ligand, FN's adhesive module III, domain 9-10 sequence through well-known integrin subset $\alpha 5 \beta 1$, quickly anchor to the matrix, form substantial focal adhesions and then conform to cytoskeletal extensions. The rapidly adherent and spreading cells obtain high proliferative capacity to reach relatively early confluence and switch to osteogenic differentiation when provided with proper biochemical cues, inducing down-regulation of genes associated with cell growth and up-regulation of genes involved in osteogenesis. While MSCs secrete several ECM molecules during multiplication and osteogenesis with time, the exogenous OC protein 
appropriately fused to Col fibrillar matrix essentially drives osteogenesis by stem cells, particularly the late stage mineralization process, by capturing / precipitating calcium ions present in culture medium or in vivo body fluid and subsequently acquiring phosphate group that enzymatically generated in the cells.

This series of stimulatory reactions in vitro and in vivo, driven by an engineered bone ECM matrix, were well demonstrated when tailored to the surface of biopolymer PCL scaffold. The current novel biomimetic matrix and the tailored scaffold will thus be potentially and extensively applicable for the repair and regeneration of bone.

Acknowledgements: This study was supported by grants from the Priority Research Centers Program (2009-0093829), National Research Foundation, South Korea. 


\section{References}

1. Hutmacher DW, Schantz JT, Lam CX, Tan KC, Lim TC. State of the art and future directions of scaffold-based bone engineering from a biomaterials perspective. J Tissue Eng Regen Med 2007;1(4):245-60.

2. Damsky $\mathrm{CH}$. Extracellular matrix-integrin interactions in osteoblast function and tissue remodeling. Bone 1999;25(1):95-6.

3. Ingram RT, Clarke BL, Fisher LW, Fitzpatrick LA. Distribution of noncollagenous proteins in the matrix of adult human bone: evidence of anatomic and functional heterogeneity. J Bone Miner Res 1993;8(9):1019-29.

4. Rammelt S, Neumann M, Hanisch U, Reinstorf A, Pompe W, Zwipp H, et al. Osteocalcin enhances bone remodeling around hydroxyapatite/collagen composites. J Biomed Mater Res A 2005;73(3):284-94.

5. Kruger TE, Miller AH, Wang J. Collagen scaffolds in bone sialoprotein-mediated bone regeneration. ScientificWorldJournal 2013;2013:812718.

6. Kim HW, Li LH, Lee EJ, Lee SH, Kim HE. Fibrillar assembly and stability of collagen coating on titanium for improved osteoblast responses. J Biomed Mater Res A 2005;75(3):629-38.

7. Krawetz RJ, Taiani JT, Wu YE, Liu S, Meng G, Matyas JR, et al. Collagen I scaffolds crosslinked with beta-glycerol phosphate induce osteogenic differentiation of embryonic stem cells in vitro and regulate their tumorigenic potential in vivo. Tissue Eng Part A 2012;18(910):1014-24 .

8. Roehlecke C, Witt M, Kasper M, Schulze E, Wolf C, Hofer A, et al. Synergistic effect of titanium alloy and collagen type I on cell adhesion, proliferation and differentiation of osteoblast-like cells. Cells Tissues Organs 2001;168(3):178-87.

9. Oldberg A, Linney E, Ruoslahti E. Molecular cloning and nucleotide sequence of a cDNA clone coding for the cell attachment domain in human fibronectin. J Biol Chem 1983;258(17):10193-6.

10. Ruoslahti E, Pierschbacher MD. New perspectives in cell adhesion: RGD and integrins. Science 1987;238(4826):491-7.

11. Aota S, Nomizu M, Yamada KM. The short amino acid sequence Pro-His-Ser-Arg-Asn in human fibronectin enhances cell-adhesive function. J Biol Chem 1994;269(40):24756-61. 
12. Lian JB, Stein GS. Development of the osteoblast phenotype: molecular mechanisms mediating osteoblast growth and differentiation. lowa Orthop J 1995;15:118-40.

13. Lee JH, Park JH, Yun YR, Jang JH, Lee EJ, Wall I, et al. Tethering bi-functional protein onto mineralized polymer scaffolds to regulate mesenchymal stem cell behaviors for bone regeneration. Journal of materials chemistry B 2013;1(21):11.

14. Chrzanowski W, Kondyurin A, Lee JH, Lord MS, Jang JH, Kim HW, et al. Nano-bio-chemical braille for cells - the regulation of stem cells using bi-functional surfaces. Adv Funct Mater (on line).

15. Jeon E, Yun YR, Kim HW, Jang JH. Engineering and application of collagen-binding fibroblast growth factor 2 for sustained release. J Biomed Mater Res A 2013.

16. Sun W, Lin H, Chen B, Zhao W, Zhao Y, Xiao Z, et al. Collagen scaffolds loaded with collagen-binding NGF-beta accelerate ulcer healing. J Biomed Mater Res A 2010;92(3):88795.

17. Abbott A. Cell culture: biology's new dimension. Nature 2003;424(6951):870-2.

18. Xu FJ, Wang ZH, Yang WT. Surface functionalization of polycaprolactone films via surfaceinitiated atom transfer radical polymerization for covalently coupling cell-adhesive biomolecules. Biomaterials 2010;31(12):3139-47.

19. Pitt CG, Marks TA, Schindler A. Biodegradable drug delivery systems based on aliphatic polyesters: application to contraceptives and narcotic antagonists. NIDA Res Monogr $1981 ; 28: 232-53$.

20. Dorj B, Won JE, Purevdorj O, Patel KD, Kim JH, Lee EJ, et al. A novel therapeutic design of microporous-structured biopolymer scaffolds for drug loading and delivery. Acta Biomater 2014;10(3):1238-50.

21. Oh SA, Kim SH, Won JE, Kim JJ, Shin US, Kim HW. Effects on growth and osteogenic differentiation of mesenchymal stem cells by the zinc-added sol-gel bioactive glass granules. J Tissue Eng 2011;2010:475260.

22. Lee JH, Park JH, El-Fiqi A, Kim JH, Yun YR, Jang JH, et al. Biointerface control of electrospun fiber scaffolds for bone regeneration: Engineered protein link to mineralized surface. Acta Biomater 2014.

23. Sun $W$, Lin $H$, Xie $H$, Chen B, Zhao W, Han $Q$, et al. Collagen membranes loaded with 
collagen-binding human PDGF-BB accelerate wound healing in a rabbit dermal ischemic ulcer model. Growth Factors 2007;25(5):309-18.

24. Wood GC, Keech MK. The formation of fibrils from collagen solutions. 1. The effect of experimental conditions: kinetic and electron-microscope studies. Biochem J 1960;75:588-98.

25. Williams BR, Gelman RA, Poppke DC, Piez KA. Collagen fibril formation. Optimal in vitro conditions and preliminary kinetic results. J Biol Chem 1978;253(18):6578-85.

26. Harris JR, Reiber A. Influence of saline and $\mathrm{pH}$ on collagen type I fibrillogenesis in vitro: fibril polymorphism and colloidal gold labelling. Micron 2007;38(5):513-21.

27. You R, Li X, Liu Y, Liu G, Lu S, Li M. Response of filopodia and lamellipodia to surface topography on micropatterned silk fibroin films. J Biomed Mater Res A 2014.

28. Guillou H, Depraz-Depland A, Planus E, Vianay B, Chaussy J, Grichine A, et al. Lamellipodia nucleation by filopodia depends on integrin occupancy and downstream Rac1 signaling. Exp Cell Res 2008;314(3):478-88.

29. Hidalgo-Bastida LA, Cartmell SH. Mesenchymal stem cells, osteoblasts and extracellular matrix proteins: enhancing cell adhesion and differentiation for bone tissue engineering. Tissue Eng Part B Rev 2010;16(4):405-12.

30. Heinegard D, Oldberg A. Structure and biology of cartilage and bone matrix noncollagenous macromolecules. FASEB J 1989;3(9):2042-51. 


\section{Figure Caption}

Figure 1. (a) Schematic representation of the $3 \mathrm{D}$ structure of functional fusion protein, (CBD)OC-FN, as illustrated using the Pymol program, and (b) Western blot showing protein expression band. (c) Turbidity curves of fibrillar collagen solution in PBS recorded with respect to incubation time at varying temperatures and Col concentrations, and (d) the turbidity curves with the incorporation of fusion protein at varying concentrations $\left(10 \% \mathrm{Col}\right.$ and $\left.25^{\circ} \mathrm{C}\right)$.

Figure 2. (a-c) SEM morphologies, showing the hybrid protein Col+(CBD)OC-FN tailored on PCL scaffolds at different magnifications. Sirius red $S$ assay shows a uniform distribution of the fusion protein throughout the scaffold (inset in (a)). Fibrillar structure of the hybrid protein networks was clearly revealed (arrowed in (c)). (d) Molecular force probe representative images also revealed the fibrillar formed surface. (e) TEM image showing the fibrillar Col and fusion protein dotted with AuNP stains.

Figure 3. (a,b) Quantification of the protein contents tethered on the scaffold surface; Col content with or without the fusion protein showing similar levels of an average of $120 \mathrm{mg}$ per scaffold sample, as quantified by Sirius red assay (a), and the fusion protein quantity present within Col fibrillar structure either adhered to the scaffold surface ('(+)scaffold') or remained in the solution ('(-)scaffold') (b). (c,d) Stability of the hybrid protein on the scaffolds measured in PBS for up to 28 days; While Col remained on the scaffolds shows a gradual release (c), the fusion protein in the Col network preserved well the fluorescence intensity (including representative images in inset, detected against anti-OC) up to the test period (d).

Figure 4. Initial adhesion behaviors of rMSCs, as assessed by the adhesion rate and gene expression. (a) Adhesion level of cells on the three different scaffolds at varying culture time points. Significantly higher cell adhesion noticed at all time points with fusion protein tailoring $(n=3)$. (b) Paxillin (PAX) mRNA expression of the rMSCs on the scaffolds by RT-PCR, at the earliest stage of cell adhesion (20 and $40 \mathrm{~min}$ ). Band intensities from three replicate experiments compared when normalized to GAPDH, showing significant stimulation with fusion protein tailoring.

Figure 5. Cell spreading behaviors examined. (a) CLSM images of cells at 2 and $4 \mathrm{~h}$ revealing nuclei 
in blue, F-actin in red and vinculin in green, and (b) the cell spreading area and periphery length of cells measured by Zen 2009 program $(n=20) .\left({ }^{*} p<0.05\right.$, PCL vs. Col; $\# p<0.05$, Col vs. OCFN).

Figure 6. rMSCs proliferation behaviors on the scaffolds during culture for up to 14 days. (a) Cell proliferation measured by MTS assay, and (b) proliferation rate (increase per day) during each time interval $(n=3)$. (c,d) Cell proliferative morphology at 14 days taken by SEM (plane view in (c) and cross-section view in (d)) and CLSM (e; nuclei in blue and F-actins in red).

Figure 7. Osteogenic differentiation and mineralization behaviors of rMSCs on the protein-tailored scaffolds. (a) ALP activity of cells measured up to 21 days $(n=5)$. (b) Cellular mineralization up to 28 daus, as quantified by ARS assay $(n=5)$. Red-stained images included. (c) EDS analysis of Ca and P elements deposited in the cellular matrix at 28 days.

Figure 8. In vivo bone regeneration examined in rat calvarium model after 6 weeks of implantation of the scaffolds. Experimental groups include empty control (-), pure PCL, with Col, and with OCFN at varying concentrations; 10CFN, 100CFN, and 1000CFN). (A) Two 6-mm circular defects generated in rat calvarium and the scaffolds implanted. (b) Micro-CT 3D reconstructed images of different sample groups, and (c) the image analyses of the newly formed bone, revealing bone volume, bone surface, and bone surface density.

Figure 9. Histological images taken at (a) low and (b) high magnifications. Enlarged images of 1000 CFN are shown representatively (H\&E stains).

Figure 10. Scheme illustrating possible series of reactions involved in the stimulatory roles played by the mimetic hybrid Col+(CBD)OC-FN matrices in MSCs. Cells specifically recognize the ligand, FN's adhesive module III, domain 9-10 sequence through well-known integrin subsets $\alpha \mathrm{v} \beta 3$ and $\alpha 5 \beta 1$, quickly anchor to the matrix, form substantial focal adhesions and then conform to cytoskeletal extensions. Rapidly adherent and spreading cells obtain high proliferative capacity to reach relatively early confluence and switch to an osteogenic differentiation when provided with proper biochemical cues. While MSCs secrete several ECM molecules like BSP and OPN during multiplication and osteogenesis with time, the exogenous OC protein appropriately fused to Col fibrillar matrix essentially drives osteogenesis of stem cells, particularly the late stage mineralization process, by capturing / precipitating calcium ions present in culture medium or in vivo body fluid and subsequently 
acquiring phosphate group that enzymatically generated in the cells. 
(a)<smiles>C[C@H]([18OH])[C@H](C)[18OH]</smiles>

(c)

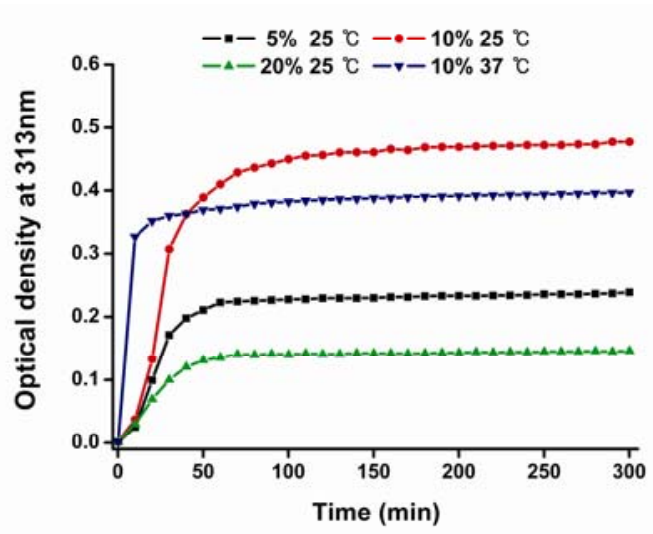

(b) $\mathrm{kDa}$

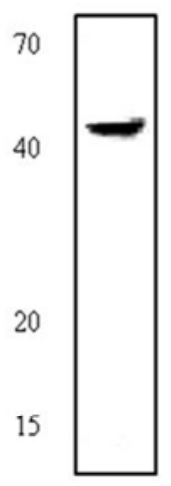

(d)

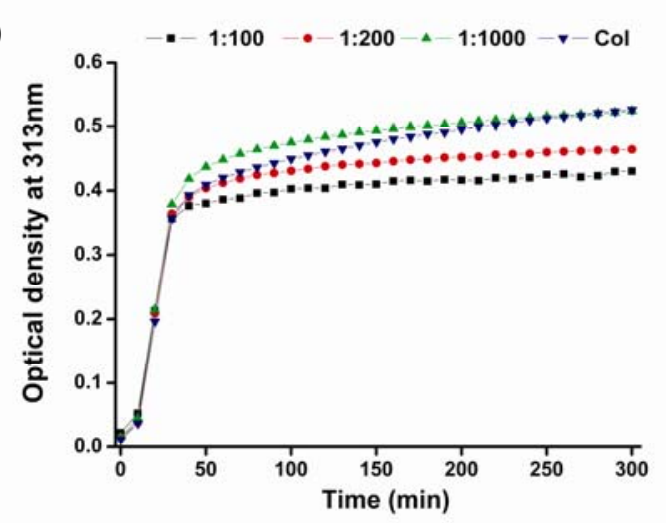

Figure 1

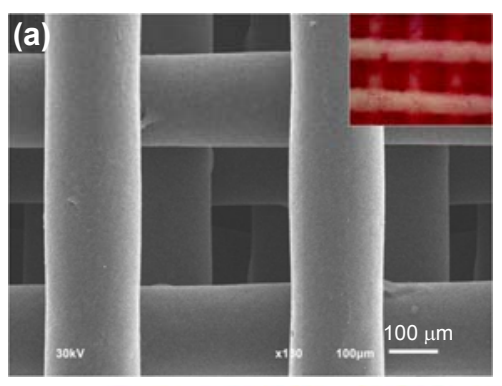

(d)

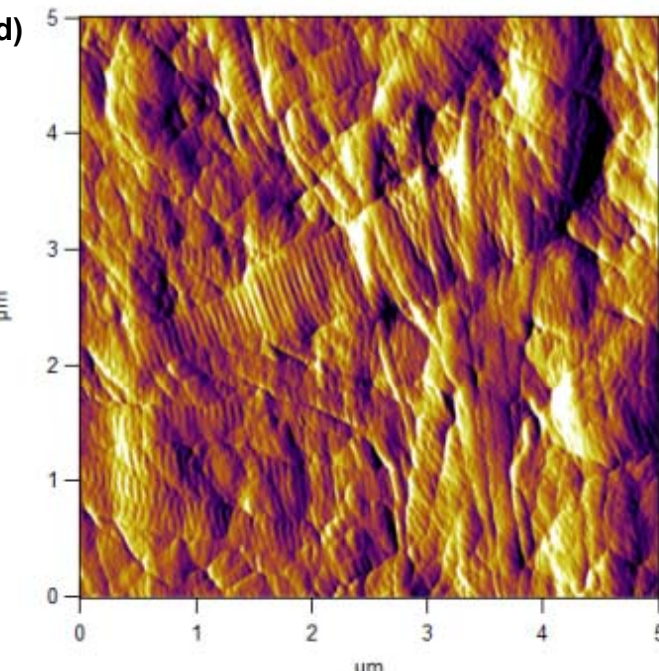

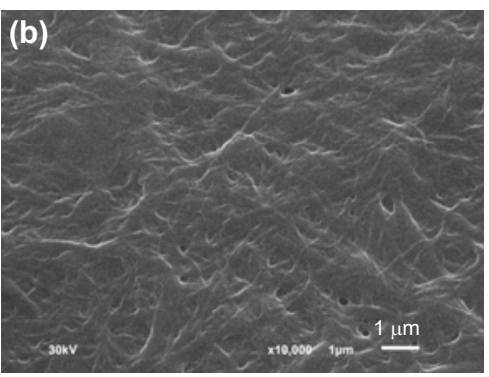

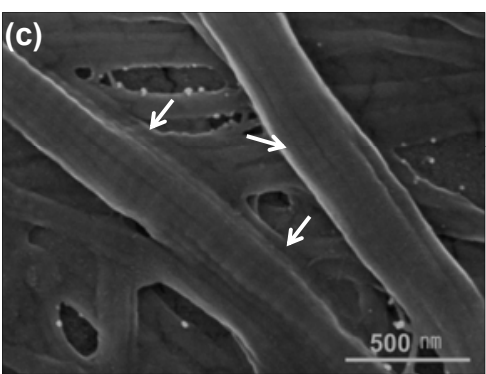

(e)

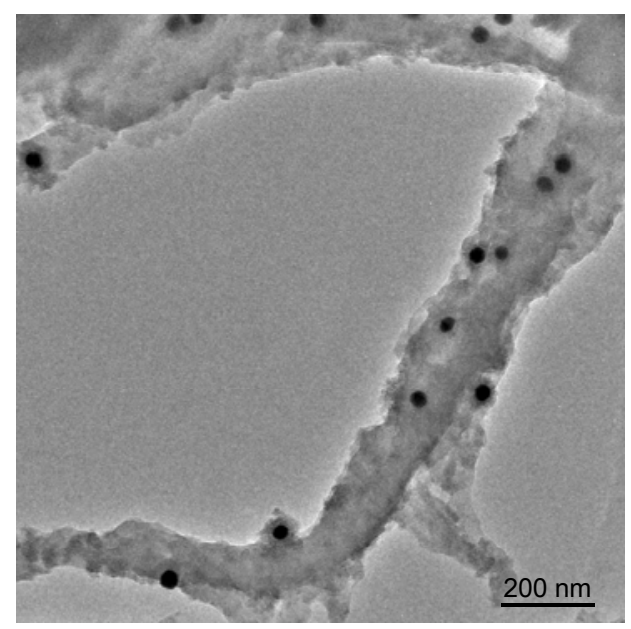



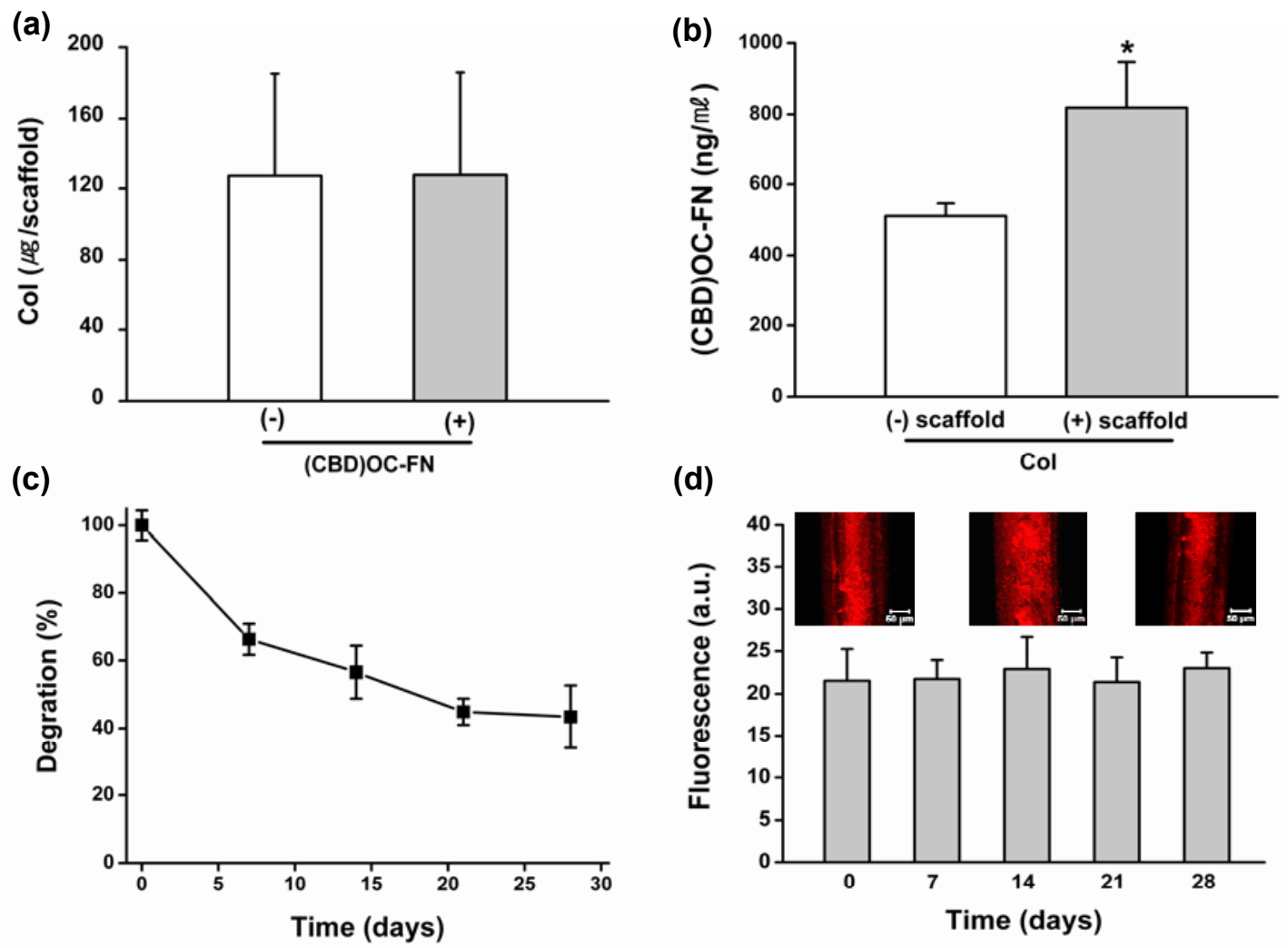

Figure 3

(a)

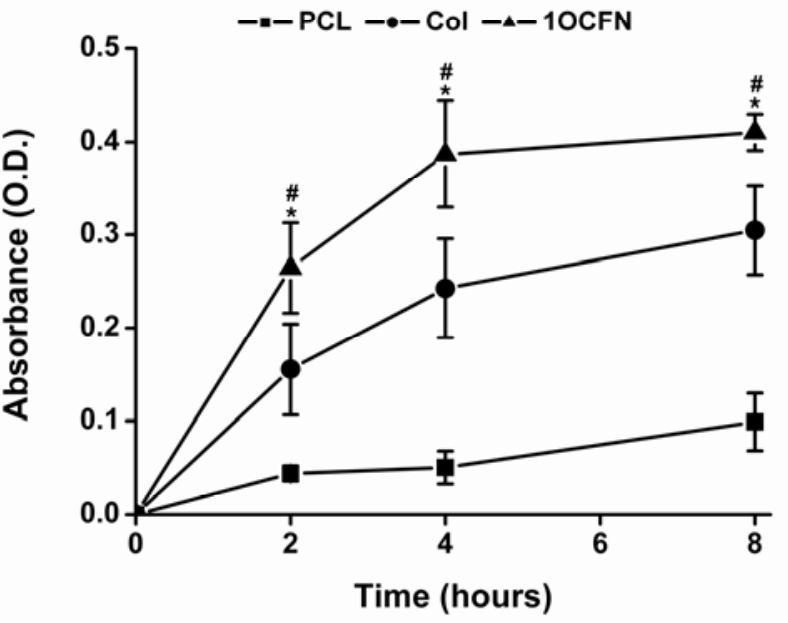

(b)

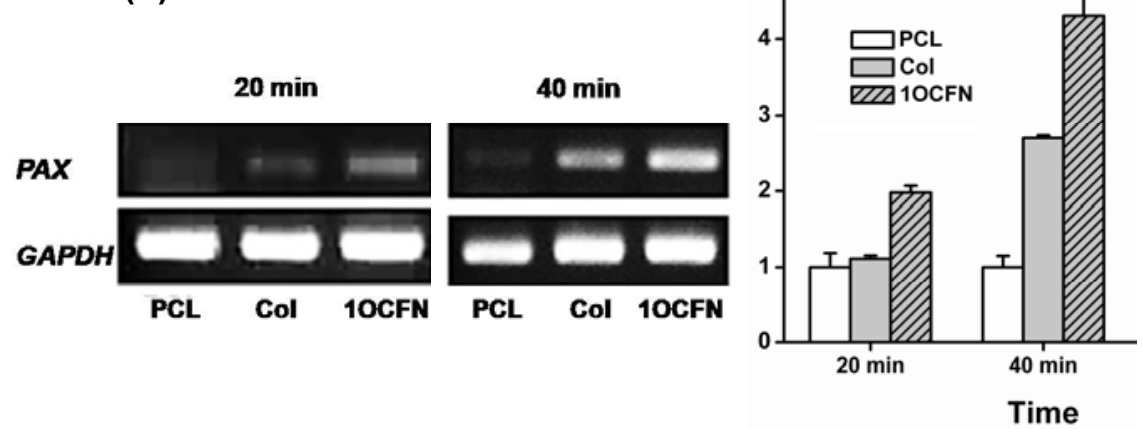




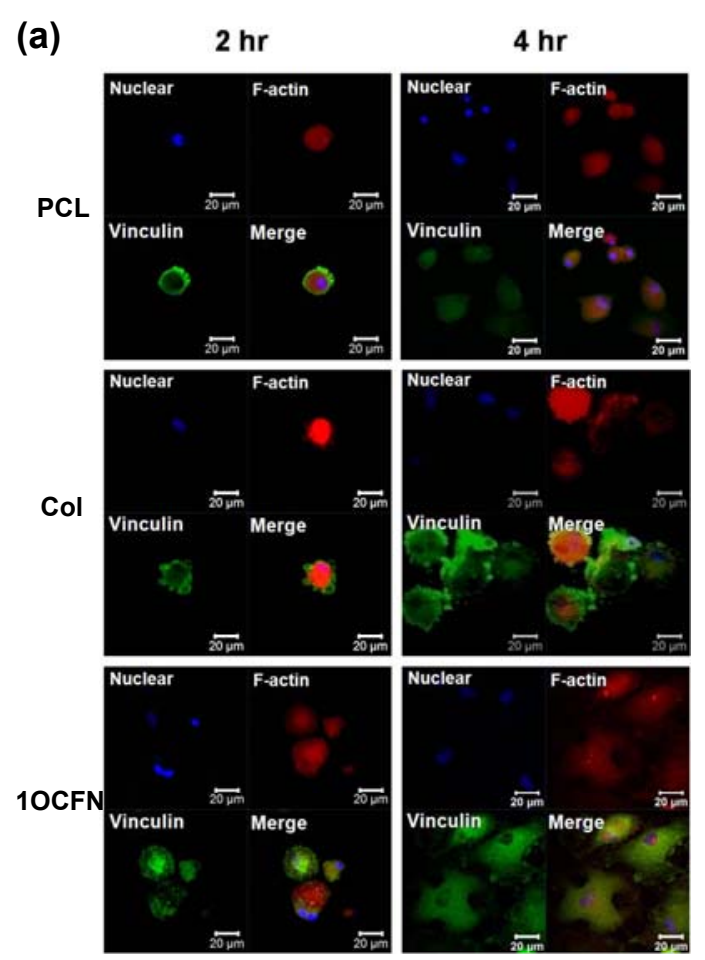

Figure 5

(b)
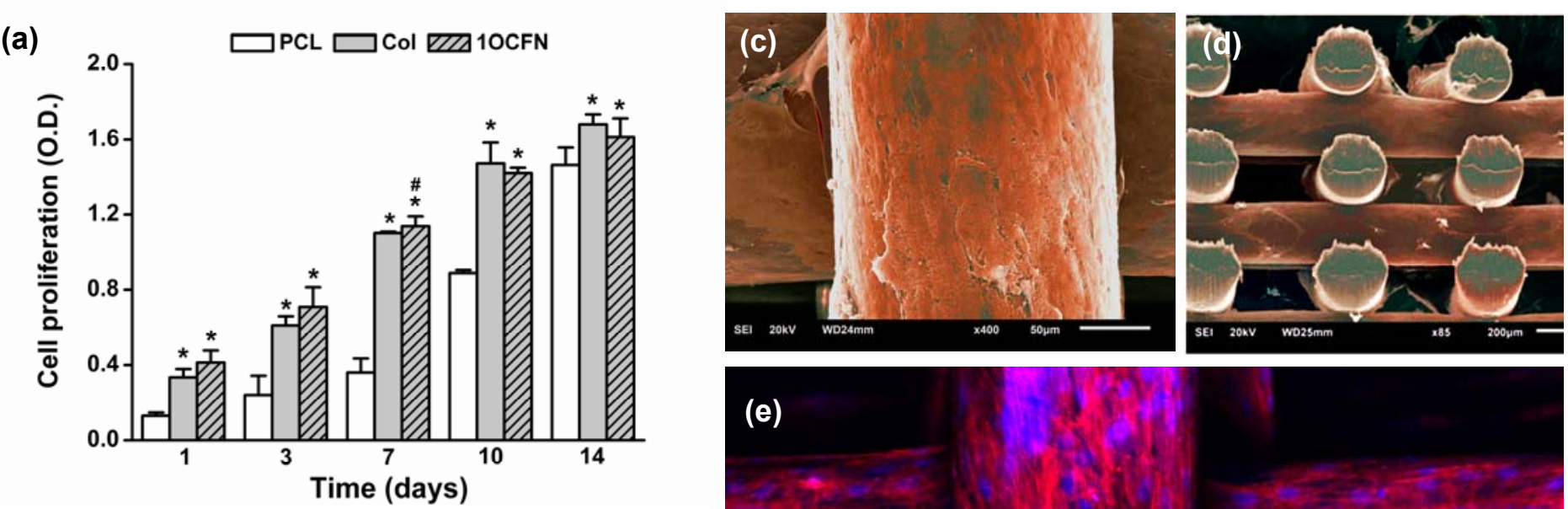

(e)

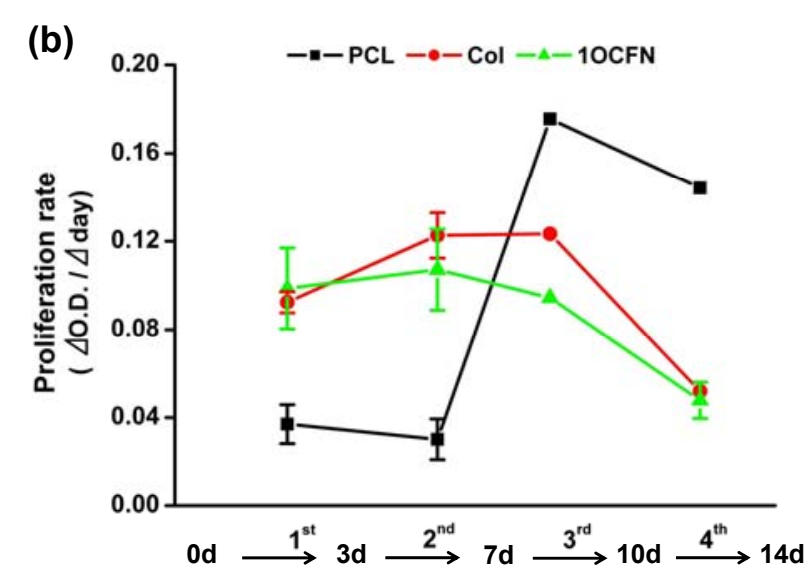


(a)

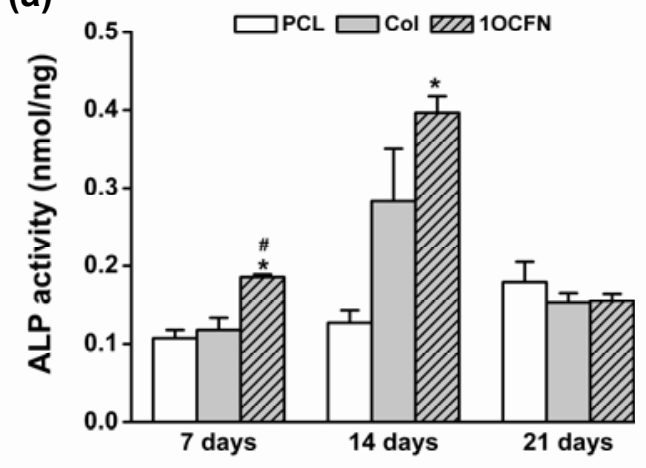

(b)

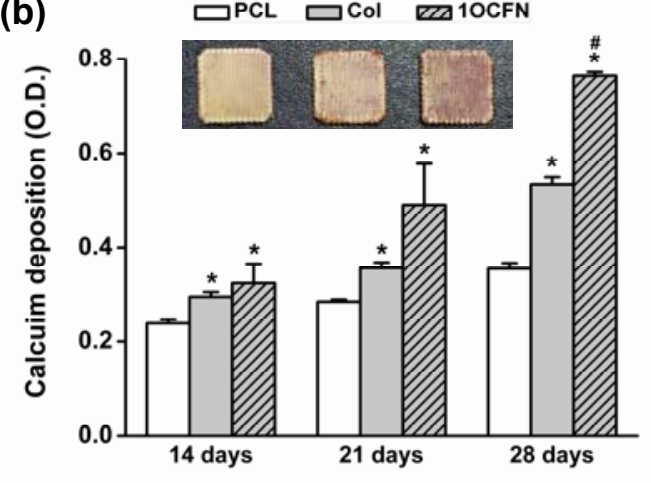

(c)

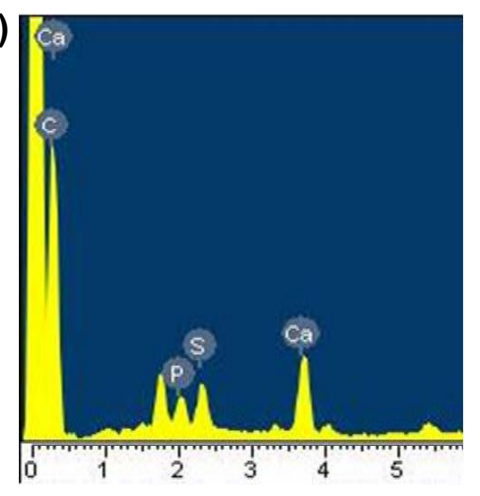

Figure 7

(b)
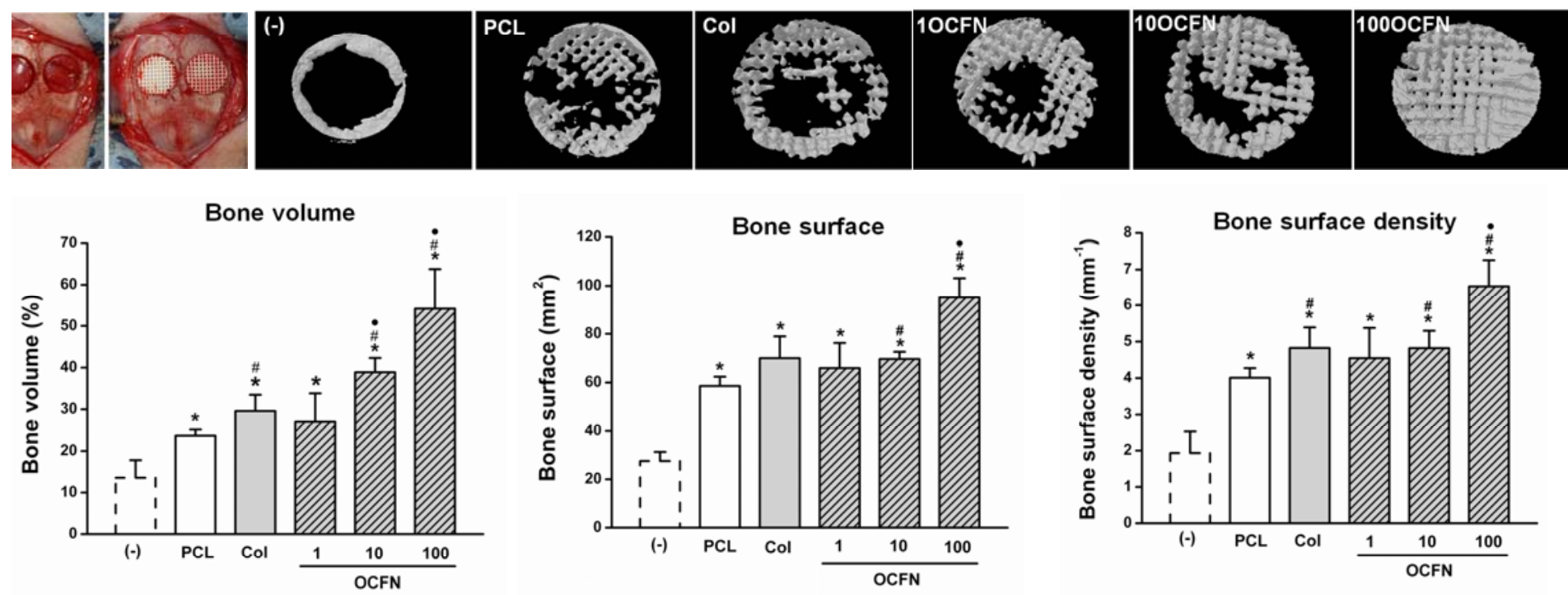
(a) (-)
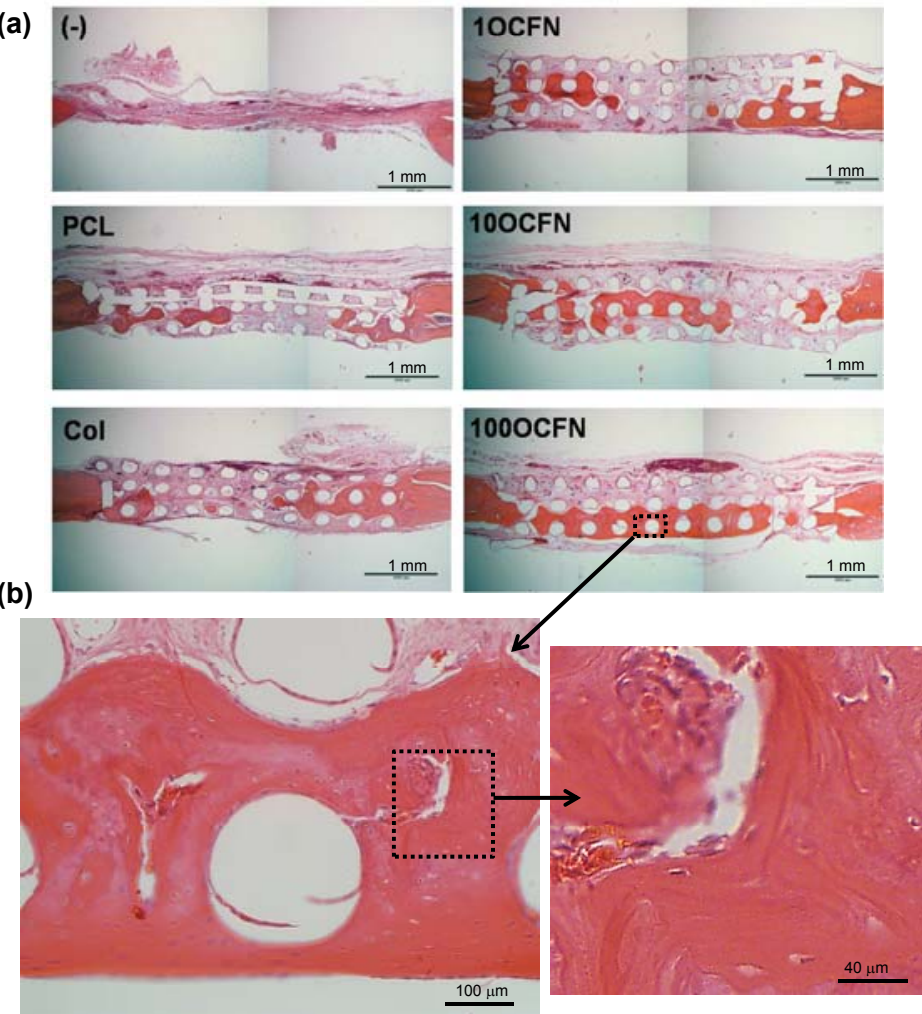

Figure 9

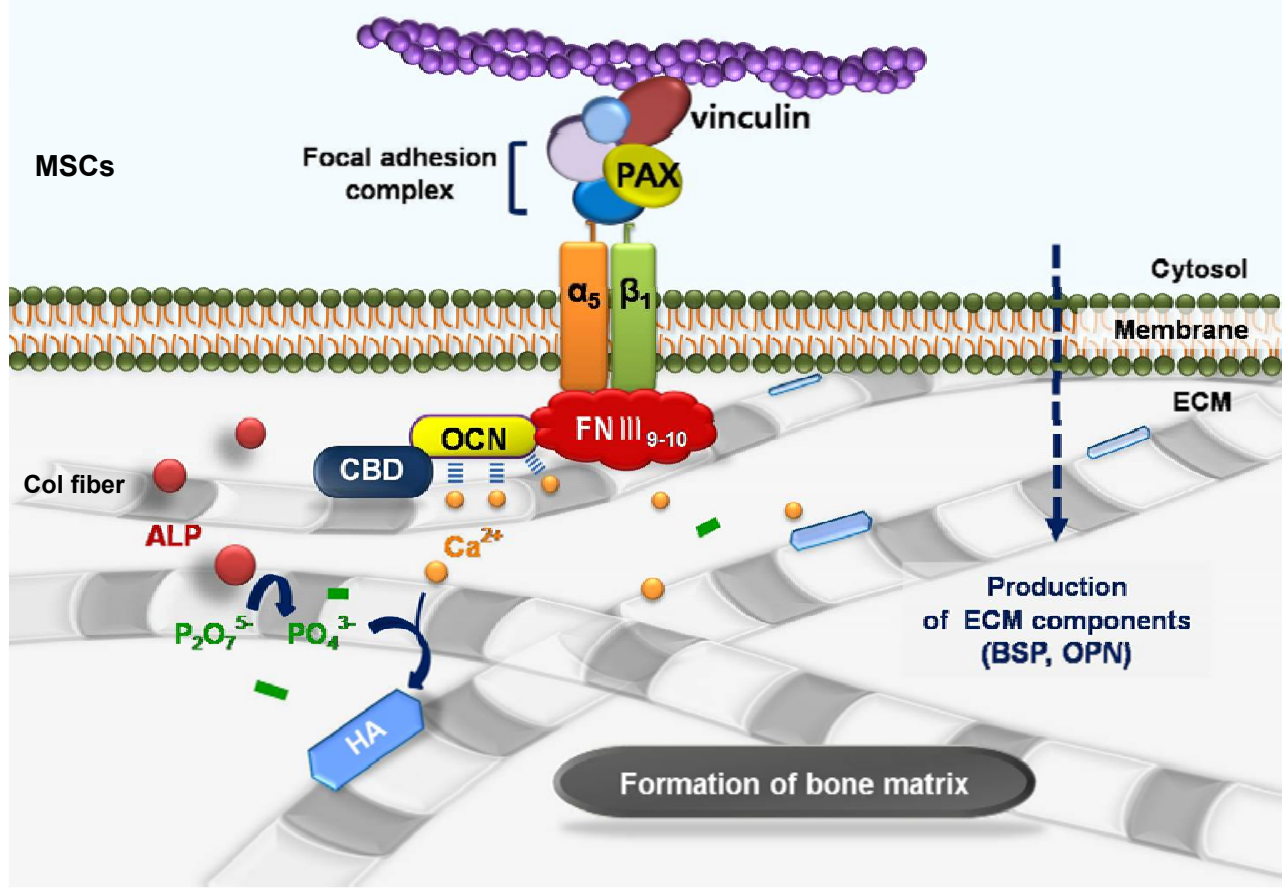

